\title{
Design and testing of instrumentation for multi-phase flow sampling and local void fraction measurement
}

\author{
Y. Yang \& D. Telionis \\ Department of Engineering Science and Mechanics, \\ Virginia Polytechnic Institute and State University, USA
}

\begin{abstract}
Instrumentation is available to obtain samples in two and three-phase flow and then measure the local void fraction as well as the size and number density of bubbles and/or particles. But in most cases, these methods interfere with the flow and bias the sampling process. We have developed an isokinetic probe that can take accurate samples without changing the sample's composition. This is achieved by aligning the probe's intake nozzle with the flow's local predominant direction and matching its internal pressure with its hydrodynamic environment, and hence equalizing the inside and outside velocities. Then the fluid sample's density is measured to calculate the local void fraction. The calibration procedure and results as well as extensive test data obtained in a bubbly-flow tunnel and flotation cells are presented and discussed.

Keywords flow sampling, void fraction measurement.
\end{abstract}

\section{Introduction}

To study the impact of the multi-phase flow characteristics it is necessary to obtain and analyze the true local samples in two- and three-phase flow. A few invasive and non-invasive techniques have been developed by scientists and engineers for research and industrial applications as reviewed by Boyer et al. [1]. A typical gas holdup meter consists of a graduate container with two open ends, two flip covers and the pneumatic actuator system. After the local fluid sample is taken, the whole device is pulled out of the tank. The gas holdup can then be read directly from the scale on the container wall. This gas holdup meter is simple and can measure the local void fraction and particle concentration 
directly. However, it is too big, which results in very low spatial resolution. A more serious problem is that it disturbs the local flow due to its large size, and hence cannot obtain accurate multi-phase fluid samples.

Gomz and Finch measured the gas dispersion in flotation cells using a gasholdup sensor, which is based on conductivity [2]. A typical conductivity probe is a hollow plastic tube with two electrodes arranged inside axially. As the name implies, the electrodes measure the conductivity of the fluid flowing through the tube. Pre-calibration is conducted in multi-phase flow to establish the relationship between the conductivity rate of the fluid and the void fraction. Comparing with the gas holdup meter, the conductivity probe is much smaller and its operation can be automated. However, the calibration procedure is very complicated. Another shortage of this device is that it is not able to obtain true samples without changing the fluid characteristics.

The McGill Bubble Viewer or its improved version is another established tool in industry. It consists of a viewing chamber and a PVC tube. The bubbles rise along the tube and then are guided into an inclined chamber. Once in the chamber, the bubbles float up and enter the upper side of the inclined window of the chamber, where a digital camera is set to capture the bubble images. Digital imaging processing technology is used to measure the bubble diameters and determine the bubble size distribution, from which the Sauter mean diameter is calculated [3].

The McGill tool is a widely-used device in the flotation industry, but this tool discriminates in its sampling and it is hard to move from point to point in the domain of interest. Moreover, allowing for bubbles and bubble/particle aggregates to rise over long elevations to the measuring location induces changes on the sample that cannot be accounted for. Additionally the McGill tool would fail entirely if it is inserted in the highly activated domains of a flotation machine, namely in the immediate neighborhood of the impeller and the stator. But this is where most of the important flotation processes are taking place, and where our observations and measurements should be concentrated. Another limitation involves cleaning the viewing chamber. As bubbles loaded with solid particles rise to the top of the view chamber, they burst and release the solid particles, which accumulate in the chamber and reduce the quality of the images.

A typical industry method for air holdup measurement is taking samples by sucking flow out from the bulk. However, if the flow is not sampled under isokinetic condition, it leads to obvious errors. Many people have stated that the aerated media must be withdrawn under isokinetic conditions, which means the multi-phase flow should be sucked in with the same speed as it approaches the sampling probe $[4,5]$. Isokinetic sampling probes have wide applications in multi-phase flow environments [6-8]. The isokinetic sampling probe working principle is described in Section 2.

In this paper we describe the development of instrumentation appropriate for exploring multi-phase flow in complex turbulent flows. The main consideration is to obtain samples without disturbing the local flow. The instruments developed provide information on the local value of the void fraction and the 
velocity components of the flow. We then describe the experiments conducted in flotation cells, present the results and discuss their significance.

\section{Instrument developments}

\subsection{Development of an isokinetic sampling probe}

Isokinetic sampling probes have been described in the literature [6-8]. But in all these cases, the alignment of the probe does not present a problem, because these probes were employed in pipe flows or other situations where the direction of the flow is known. In the problems we need to explore here the flow is very complex and the direction of the flow is not known. This requires that a method must be devised to align the probe with the local direction of the velocity vector. Another requirement is that the flow velocities inside and outside the probe is the same, so that isokinetic conditions are established. We devised a method that could guide the operator to make these adjustments for accurate measurements.

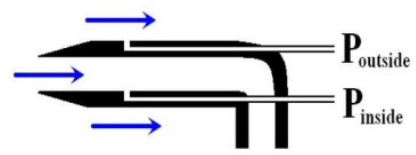

Figure 1: $\quad$ Sketch of isokinetic sampling probe.

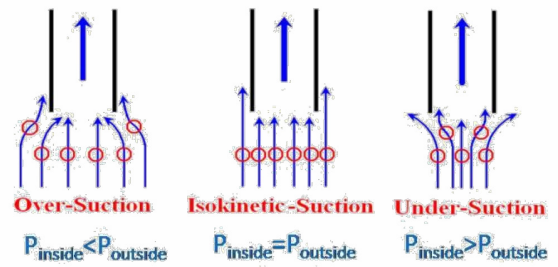

Figure 2: $\quad$ Working principle of isokinetic sampling probe.

The isokinetic principle is described schematically in Figure 1. The outlet of the isokinetic sampling probe is connected to a suction pump. Multi-phase flow is sucked through the inlet with the probe positioned parallel to the main stream. If the velocity in the nozzle is equal to the velocity of the surrounding stream, the main flow is not disturbed. Only when this condition is fulfilled, we can state that the sampling is isokinetic as shown in Figure 2. Sampling and accurate measurements can be obtained only in isokinetic suction conditions. This means that the probe should be aligned with the local flow, and the sampled flow must enter the probe without linear or angular acceleration. In sampling bubbly flow, over-suction gives higher void fraction readings while under-suction gives lower readings. In water-particle flow, the opposite is true: over-suction results in lower particle concentration readings while under- suction results in higher readings. 
The probe we developed has two pressure taps, one inside the probe and another outside the probe, as shown schematically in Figure 1. The operator adjusts the suction pump so that $\mathrm{p}_{\text {outside }}$ is equal to $\mathrm{p}_{\text {inside }}$.

To obtain the conditions of isokinetic sampling the probe must first be aligned with the local direction of the flow. The innovating feature of the probe we developed consists of the following. Four pressure ports have been placed on the outside wall at circumferential locations 90 degrees apart from each other. When the flow is aligned with the flow the four pressures are equal to each other. To align the probe with the flow, the operator must adjust pitch and yaw until this condition is met.

\subsection{Flow visualization of isokinetic conditions}

To confirm the working principle described above, two-dimensional flow visualization experiments were set up and run. Two pieces of Plexiglas plates were placed parallel to each other to form a two-dimensional channel. This channel was set in the middle of a closed-circuit water tunnel, which includes tubes, valves and a pump. A sparger was set near the channel's inlet to generate air bubbles. This system can generate uniform bubbly flow in the twodimensional channel. A two-dimensional isokinetic sampling probe was fabricated and installed in the middle of a test section aligned with the flow. The outlet of the probe was connected to a positive displacement pump, while the two pressure ports were connected to a pressure manometer to monitor the pressure difference. During the experiments, the suction rate of the positive displacement pump was adjusted to obtain different operation conditions, i.e. under-suction, over-suction, isokinetic suction and non-suction. The light source was set behind the two-dimensional channel to illuminate the test section, while a camera was set on the opposite side to record the flow pattern. A low shutter speed was chosen to allow the visualization of bubble trajectories.

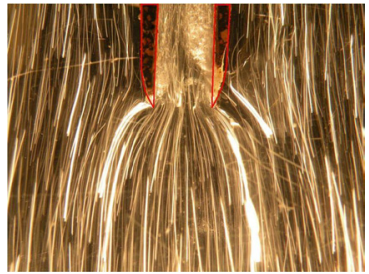

Over-suction

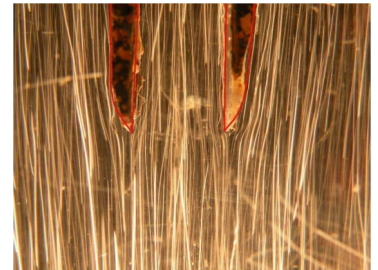

Isokinetic-suction

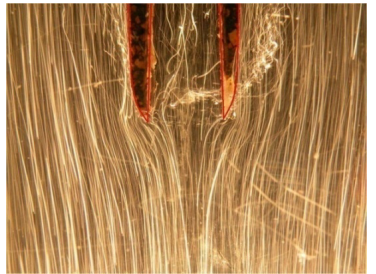

Under-suction

Figure 3: $\quad$ Flow visualization under different operation conditions.

The visualization results are shown in Figure 3. As described above, the bright lines are the trajectories of air bubbles. For the case of over-suction, bubbles are shown populating the entrance to the probe, leaving the flow right outside the probe with low bubble content. While the under suction case has the opposite effect. Less bubbles enter the sampling probe, which make the void fraction inside lower than the local flow. The non-suction case could be regarded 
as an extreme condition of under suction. From the photo we can see that no bubbles enter the sampling probe.

Over-suction and under-suction happen when the pressure in the sampling tube is lower or higher than the ambient pressure. This type of segregation is explained as follows: Over-suction and under-suction result in sharp curving of the streamlines near the entrance of the sampling tube. Curved streamlines establish cross-flow pressure gradients, with the lower pressures towards the concave side of the streamlines. Thus a radial gravity field is established. Light materials like bubbles are pushed towards the concave side, just as if they are sucked towards the center of a vortex. But heavy materials are jettisoned towards the convex side of the streamlines. These phenomena are theoretically and experimentally verified. If the streamlines are directed along the axis of the tube with no curving, then there will be no discrimination of light or heavy materials that are transported with the flow. These conditions can be achieved first by aligning the probe with the flow, and then securing that the pressure inside the tube is the same with the pressure outside the tube.

\subsection{Void fraction analyzer}

One of the multi-phase flow properties of interest that can be obtained by isokinetic sampling is the local void fraction. To determine the local void fraction, we devised the system shown in Figure 4, which was designed, constructed and tested. Driven by a pump with adjustable head, the fluid passes along an ascending and a descending part of a tube, and the static pressures are measured at four pressure ports. Pressure differences can then return the air fraction according to the following derivation which is based on the energy equation.

$$
\begin{gathered}
P_{1}-P_{2}=\rho_{\text {mix }} g H+P_{\text {loss }} \\
P_{3}-P_{4}=\rho_{\text {mix }} g H-P_{\text {loss }} \\
P_{1}-P_{2}+P_{3}-P_{4}=2 \rho_{\text {mix }} g H g H \\
\rho_{\text {mix }}=\frac{P_{1}-P_{2}+P_{3}-P_{4}}{2 g H} \\
\text { void fraction }=1-\frac{\rho_{\text {mix }}}{\rho_{\text {water }}}=\frac{P_{1}-P_{2}+P_{3}-P_{4}}{2 \rho_{\text {water }} g H}
\end{gathered}
$$

Note that with a constant inner diameter of the pipe, the velocity does not vary along the length of the tube, and thus these relationships are just hydrostatic. In this design, wall friction is compensated using the two sections of

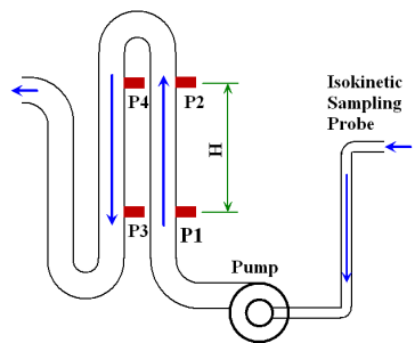

Figure 4: Local void fraction measurement system. 
the pipe, namely the ascending and descending sections where the flow is first opposite to and then points in the direction of gravity. This device can collect data automatically, and works for both two-phase flow and three-phase flow.

It is essentially a densitometer that can operate on line with the fluid flow. It is not necessary to carry out static measurements. One such densitometer module is needed for two-phase flow. But a design modification can be used to obtain both void fraction and particle concentration.

The isokinetic sampling system can obtain the true sample from the target region. However, for the void fraction measurement, the bubble size of the sample may change due to the difference of elevation between the sampling and measurement points. Static pressure correction is implemented during data analysis as described by Yang [9].

\subsection{Uncertainty analysis for the void fraction measurements}

The local void fraction calculation is based on pressure measurements as shown in Equation 5. According to the error propagation principle, the uncertainty of the local void fraction can be written as follows:

$$
\begin{gathered}
\delta V F=\frac{1}{2 \rho_{\text {water }} H}\left(\delta P_{1}+\delta P_{2}+\delta P_{3}+\delta P_{4}\right) \\
\delta V F=\frac{1}{2 \rho_{\text {water }} g H} \sqrt{\left(\delta P_{1}\right)^{2}+\left(\delta P_{2}\right)^{2}+\left(\delta P_{3}\right)^{2}+\left(\delta P_{4)}\right)^{2}} \\
\frac{\delta V F}{V F}=2 \frac{\delta P}{P} \times 100 \%
\end{gathered}
$$

Here $V F$ is the void fraction and $\delta P$ is the uncertainty of the pressure transducer reading. As we can see, the uncertainty of the void fraction measurement is directly related to the uncertainty of the pressure sensors. The error associated with the pressure sensor used in the experiments is $\pm 0.25 \%$ of the full scale.

\subsection{Calibration of the isokinetic sampling probe}

To test and calibrate the isokinetic sampling probe system, a vertical bubbly flow tunnel and a horizontal multi-phase jet tunnel were designed and built. These tunnels consist of pump, pipe line and air sparger system and can generate bubbly flows with known and repeatable laboratory conditions.

Since the high-speed jet tunnel can be run in both single-phase flow and multi-phase flow conditions, it can be used to simulate the flows found in the high-speed domains of flotation machines. This would be adequate for the testing and calibration needs, but this facility does not offer optical access to the domain of interest. Such access is available in the vertical bubbly-flow tunnel. Moreover, by controlling the air flow rate and surfactant concentration we can generate in the vertical bubbly-flow tunnel much larger bubbles that are common in flotation machines. 
The isokinetic sampling probe and the densitomer were tested in lab. The isokinetic sampling probe was aligned with the flow in both the vertical bubbly flow tunnel and horizontal high speed jet tunnel. Air and water supply rates were measured by independent flow meters. In terms of these quantities we calculate the void fraction and were able to also measure it directly. The results are presented by Yang [9] and show that the isokinetic system provides accurate measurements with excellent repeatability. The deviation is less than $2 \%$.

To show how important the isokinetic sampling is to the void fraction measurement, some tests were conducted under non-isokinetic conditions. The effect of the suction rate and inclined angle were examined as shown in Figure 5. These results indicate that the errors in proper setting of the suction rate and the probe alignment lead to serious measurement errors. For the under-suction case, the reading of local void fraction is also lower because less number of bubbles get into the isokinetic sampling probe as discussed above, while for the oversuction case, we always obtain higher readings. Errors in the probe alignment always return higher readings. These tests indicate that it is very important to align the probe with the flow, and match the suction rate to insure that the suction velocity is equal in magnitude to the ambient flow velocity. However, from the plots we can also see that minor departures in alignment and suction rates do not affect the reading very much.
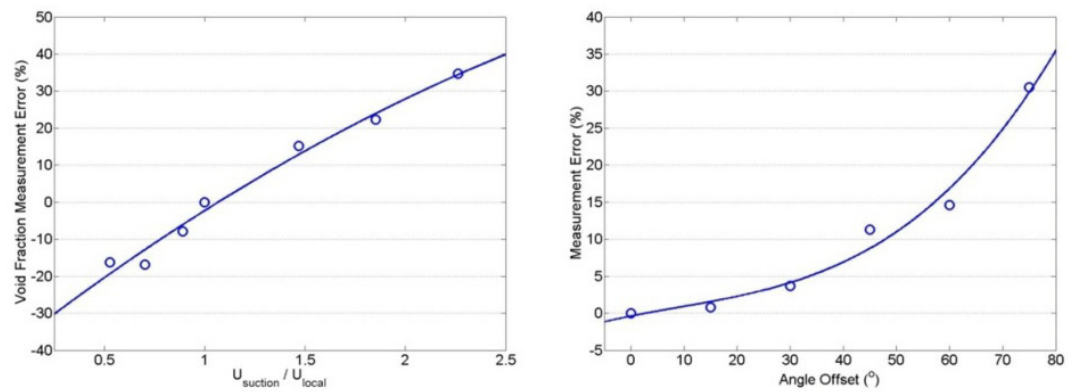

Figure 5: Influence of suction rate (left) and offset angle (right).

\section{Experiment layout}

Froth floatation recovery rate depends on a wide range of complex factors. One of the most important is the gas dispersion characteristics within the flow. Our efforts were directed mostly in flotation research. But flotation flows combine a wide range of multi-phase flow features, and offer great opportunities for the development of instrumentation. The local void fraction has direct influence on the flotation efficiency. So far it has not been possible to measure local values of void fraction. The probe we developed was employed to conduct such measurements in a $6-\mathrm{m}^{3}$ Dorr Oliver froth flotation machine. Figure 6 shows the rotor-stator assembly as well as the coordinate system that we used. The rotor has 6 blades while the stator has 16 blades. The origin of the coordinate system - 
was set at the middle of the rotor bottom. All the dimensions are normalized by the rotor diameter $\mathrm{D}_{\mathrm{i}}$.
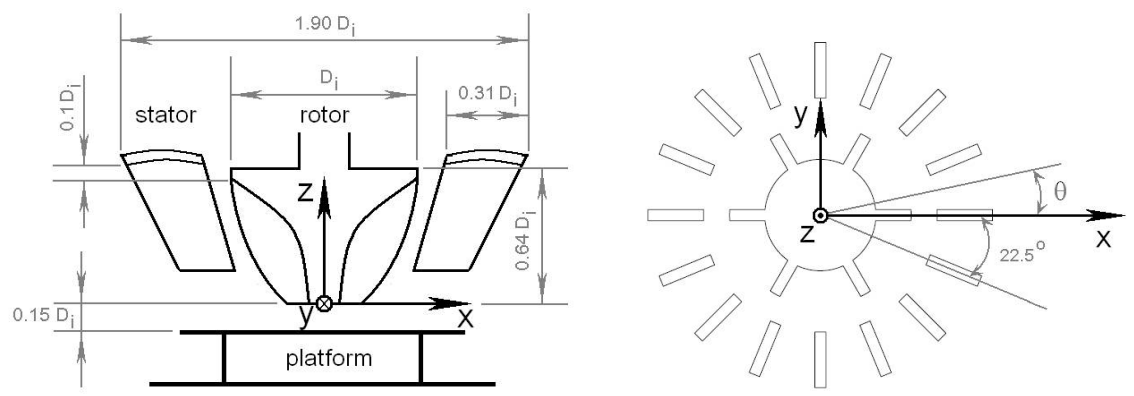

Figure 6: $\quad$ Rotor-stator assembly, side view (left) and top view (right).

As discussed above, two steps are required before samples can be obtained using the isokinetic sampling probe. First the probe axis must be aligned with the local direction of the flow. Then the suction velocity must be matched with the local flow. Both steps are guided by pressure measurements. Aligning the probe with the flow requires that these four pressures measured on the side of the probe are equal to each other. Once alignment is achieved, then the suction level must be adjusted. Now two pressure measurements are needed, one on the inside wall of the probe and another on the outside wall. Since the flow is aligned with the flow, matching the inside pressure with the outside pressure insures that the inside velocity is equal to the outside velocity and isokinetic conditions have been achieved.

To facilitate the alignment with the flow, the probe is mounted on a gimbal mechanism. This mechanism can adjust the pitch and yaw angle of the probe. The gimbal mechanism is bolted to a pole which is mounted to a traversing system. By twisting the pole, the yaw angle can be adjusted. The pitch angle is controlled through a flexible drive shaft and thread. Currently all these processes are carried out manually, but automation is possible if a large number of measurements are needed.

\section{Results and discussion}

The tools developed and described above were employed in measurements conducted in industrial flotation machines. The probe was set in the jet region at $\mathrm{x}=1.260 \mathrm{D}_{\mathrm{i}}, \mathrm{y}=-0.071 \mathrm{D}_{\mathrm{i}}$ and $\mathrm{z}=0.653 \mathrm{D}_{\mathrm{i}}$ (see Figure 6 for nomenclature). The rotor's tip velocity was set at $6.4 \mathrm{~m} / \mathrm{s}$ and the concentration of the surfactant (MIBC) was $14 \mathrm{ppm}$, while the air pumping flow rate was varied to study its influence on the local void fraction. In Figure 7 we present the local air fraction measured in the jet region as a function of the air flow rate supplied to the machine. It appears that the relationship is linear, indicating that at the point of 


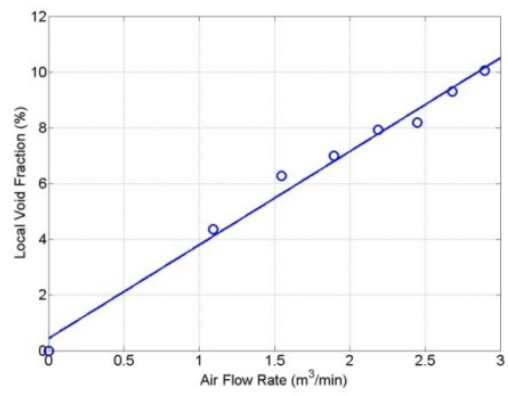

Figure 7: $\quad$ Effect of air flow rate.

measurement, the local air fraction is directly related to the supplied air flow rate.

The probe was set in the jet region at $\mathrm{x}=1.260 \mathrm{D}_{\mathrm{i}}, \mathrm{y}=-0.071 \mathrm{D}_{\mathrm{i}}$ and $\mathrm{z}=0.653 \mathrm{D}_{\mathrm{i}}$. The air pumping flow rate was set to be $2.63 \mathrm{~m}^{3} / \mathrm{min}$ and the concentration of the surfactant-MIBC- was $14 \mathrm{ppm}$, while the rotor's tip velocity was varied to study its influence on the local void fraction. Figure 8 presents measured void fractions. In Figure 8, left frame we present void fractions for varying blade tip velocities with fixed air rates. It is interesting to see that when the rotor's tip velocity is lower than $3 \mathrm{~m} / \mathrm{s}$, almost no air reaches the point of measurement. Apparently buoyancy drives the air up immediately downstream of the blade edges and through the rotor-stator gap. As the rotor's tip velocity increases, we see that for low tip velocities, the void fraction increases almost linearly, but this trend changes and the increases are gradual, until at high tip velocities there is very small influence of this quantity on the void fraction. This indicates that increasing the speed further will not be accompanied with higher void fractions, which means for the air dispersion purpose, this level of tip velocity and corresponding power input is optimal. It is possible that at high speeds, air is dispersed in a wider area and is not captured by our probe at the specific location of $\mathrm{x}=1.260 \mathrm{D}_{\mathrm{i}}, \mathrm{y}=-0.071 \mathrm{D}_{\mathrm{i}}$ and $\mathrm{z}=0.653 \mathrm{D}_{\mathrm{i}}$.

The probe was set in the jet region where $x=1.260 D_{i}, y=-0.071 \quad D_{i}$ and $\mathrm{z}=0.653 \mathrm{D}_{\mathrm{i}}$. The air pumping flow rate was set to be $2.63 \mathrm{~m}^{3} / \mathrm{min}$ and the rotor's
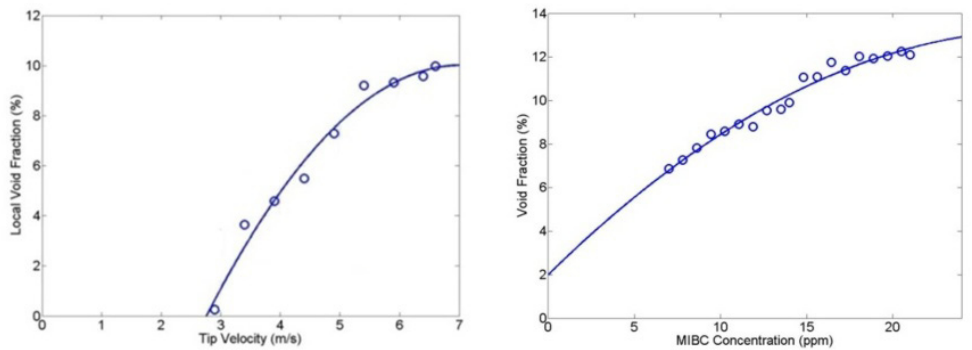

Figure 8: $\quad$ Effect of rotor tip velocity (left) and MIBC concentration (right). 
tip velocity was set to be $6.4 \mathrm{~m} / \mathrm{s}$ while the concentration of the surfactant MIBC - was varied to study its influence on the local void fraction. The results shown in Figure 8, right indicate that MIBC mixtures not only affect the size of the bubbles but also dramatically affect the void fraction.

Local void fractions at different elevations were obtained in the $6-\mathrm{m}^{3}$ machine as shown in Figure 9 (left). These measurements were obtained by traversing the probe along a horizontal line covering the space downstream of a stator. The point $\theta=0$ is along the radial extension of a stator blade. Positive angle values mean that the probe is downstream of the separated region of the stator blade (leeward side), and negative values is the attached flow side, namely the windward side. The results indicate that the air is dispersed on both the active and the inactive zones. The jet region has relatively high local void fraction since the air bubbles are brought out by the jet stream. However, the void fraction distribution is pretty uniform along the horizontal lines. The local void fraction in the dead zone is only around $10 \%$ less than the jet region.

In Figure 9 (right), void faction measurements obtained along a vertical line are presented crossing the jet where $y / D_{i}=-0.071$. This Figure indicates that the void fraction increases with the depth. Apparently buoyancy of very small bubbles is very small, and their tendency to rise is overcome by the convective action of the stream, even in the regions where the flow velocity is small.
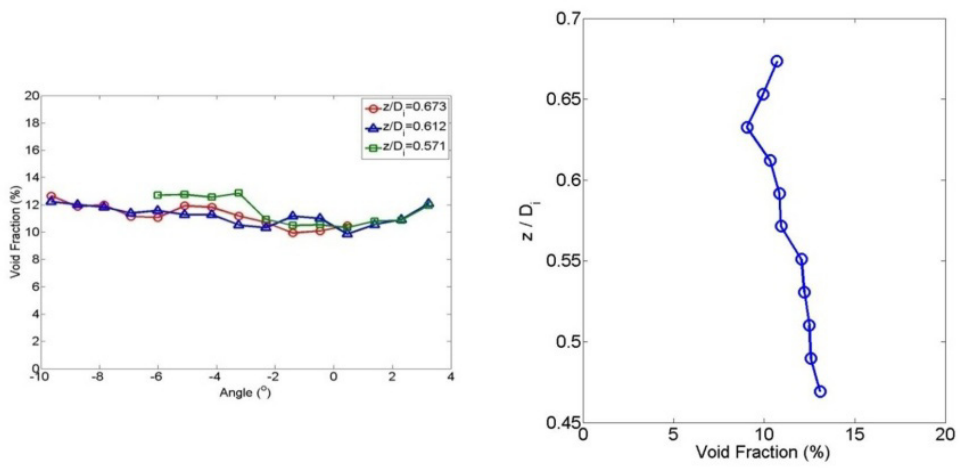

Figure 9: $\quad$ Void fraction along horizontal (left) and vertical (right) lines.

The isokinetic sampling probe was set in the jet $\left(y / D_{i}=-0.071, z / D_{i}=-0.653\right)$ to measure the local void fraction under different operation condition. At the same time, the rising level of the water free surface was measured to calculate the global air holdup. The rotor tip velocity was set as $6.4 \mathrm{~m} / \mathrm{s}$ while the air flow rate varied. Figure 10 shows that the global air holdup is always higher than the local void fraction in the jet region. This confirmed our previous conclusion: the highest local void fraction is not in the jet region. Moreover, we observe that the local value of the void fraction in the jet region follows consistently the global value of air hold up. 


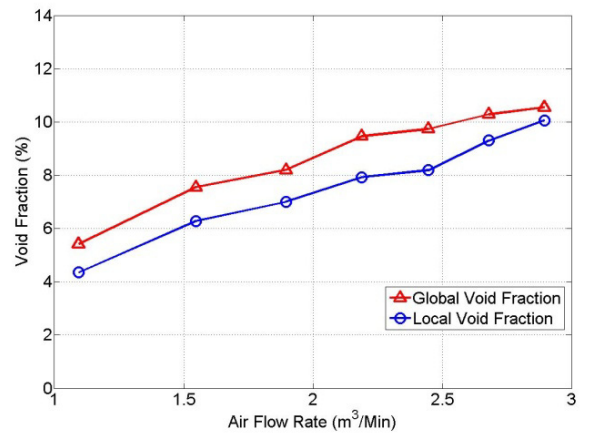

Figure 10: Comparison of overall air holdup and local void fraction.

\section{Summary and conclusions}

An isokinetic sampling system was invented for multi-phase flow characterization. The isokinetic probe has a pressure port inside to measure the inside static pressure. Four pressure ports are arranged on the stem on the circumferential direction to measure the outside static pressure. The four pressure ports are used to align the probe with the local flow by detecting the flow direction, which is a unique feature of the device.

This isokinetic probe system was used to take true samples of the bubbly flow in a flotation tank. The samples were then analyzed to obtain the local void fraction continuously. The system can also be extended to conduct local solid concentration measurements. Local void fraction measurements show that the air bubbles are carried by the stream and dispersed to the bulk uniformly, even in the inactive zone. The local void fraction measurement provides the information to evaluate the air dispersion ability of the tank. It also provides the flow density information, which is used for velocity measurement by using multi-hole probes.

\section{References}

[1] Boyer, C., Duquenne, A.-M., Wild, G., Measuring techniques in gas-liquid and gas-liquid-solid reactors. Chemical Engineering Science, 57(16): p. 3185-3215, 2002.

[2] Gomez, C.O., Finch, J. A., Gas diserprsion measurements in flotation cells. International Journal of Mineral Processing, 84: p. 51-58, 2007.

[3] Schwarz, S., Alexander, D., Gas dispersion measurements in industrial flotation cells. Minerals Engineering, 19: p. 554-560, 2006.

[4] Greaves, M., Kobbacy, K., Measurement of bubble size distribution in turbulent gas-liquid dispersion. Chemical Engineering Research and Design, 62: p. 3-12, 1984.

[5] Sherwood, J.D., Bubble collection by a point sink in a uniform flow. International Journal of Multiphase Flow, 29: p. 621-627, 2003. 
[6] Boothroyd, R.G., An anemometric isokinetic sampling probe for aerosols. Journal of Scientific Instruments, 44: p. 249-253, 1967.

[7] Zhang, G.J., Ishii, M., Isokinetic sampling probe and image processing systems for droplet size measurement in two-phase flow. International Journal of Heat and Mass Transfer, 38(11): p. 2019-2027, 1995.

[8] Dennis, R., Samples, W. R., Anderson, D. M., Silverman, L., Isokinetic sampling probes. Industrial and Engineering Chemistry, 49(2): p. 294-302, 1957.

[9] Yang, Y, Experimental study of multi-phase flow hydrodynamics in stirring tanks. PhD Dissertation, Virginia Polytechnic Institute and State University, 2011. 